\title{
Finite Element Modeling of Brain Tumor Mass-Effect from 3D Medical Images
}

\author{
Ashraf Mohamed ${ }^{1,2}$ and Christos Davatzikos ${ }^{1,2}$ \\ 1 CISST NSF Engineering Research Center, \\ Department of Computer Science, Johns Hopkins University \\ 2 Section for Biomedical Image Analysis, Department of Radiology, \\ University of Pennsylvania School of Medicine \\ \{ashraf, christos\}@rad.upenn.edu
}

\begin{abstract}
Motivated by the need for methods to aid the deformable registration of brain tumor images, we present a three-dimensional (3D) mechanical model for simulating large non-linear deformations induced by tumors to the surrounding encephalic tissues. The model is initialized with $3 \mathrm{D}$ radiological images and is implemented using the finite element (FE) method. To simulate the widely varying behavior of brain tumors, the model is controlled by a number of parameters that are related to variables such as the bulk tumor location, size, mass-effect, and peri-tumor edema extent. Model predictions are compared to real brain tumor-induced deformations observed in serial-time MRI scans of a human subject and 3 canines with surgically transplanted gliomas. Results indicate that the model can reproduce the real deformations with an accuracy that is similar to that of manual placement of landmark points to which the model is compared.
\end{abstract}

\section{Introduction}

Accurate deformable registration of $3 \mathrm{D}$ brain tumor images into a common stereotactic space is needed for the construction of brain tumor atlases. Such atlases will be useful in planning neuro-surgical tumor operations and therapeutic approaches by linking the functional and structural information provided by multi-modality images to variables such as tumor size, grade, subsequent progression, therapeutic approach and outcome [1-4].

Currently available image registration approaches applied to a register a normal brain atlas and a tumor-bearing image have limited accuracy in and around the tumor area. This is due to the inability of these approaches to account for topological differences between the two images, severe deformation in the vicinity of the tumor, and the confounding effects of edema and tumor infiltration. Approaches that introduce a small tumor "seed" in the atlas brain and rely on image information to subsequently adapt the atlas to the subject's images produce acceptable registration results for some tumor cases, however, patients with large tumors and substantial brain tissue deformation still present a major challenge 3,4 . For such cases, the lack of a physically realistic model of brain tissue deformation, derails the image matching process and causes the deformable registration to fail near the tumor. 
In this paper, a 3D finite element $(\mathrm{FE})$ model of the mass-effect of brain tumors is presented. The model is controlled by a number of parameters that are related to variables such as the tumor size, location, and peri-tumor edema extent. The exact value of these parameters for a particular tumor patient can only be found within the context of inverse problem solving based on the patient's images. The goal behind the model presented here is not to predict tumor growth, or the mass-effect for a particular tumor patient, but rather to use this model for generating a large number of brain anatomies deformed by simulated tumors for a range of the model parameters. These simulated anatomies will act as training samples for a statistical estimator of the model parameters for particular brain tumor cases. Once the model parameters are estimated, they can be used to introduce a tumor in the atlas and simulate the associated mass-effect, prior to applying a deformable image registration approach to the two images. This paper is dedicated to presenting the details of a mechanical model for tumor mass-effect and its partial validation via a number of real tumor cases. The integrated approach for image registration is described in [5].

In contrast to previous similar models that used 2D simulations and included only bulk tumor mass-effect [1, 2, 6], the model presented here is fully 3D and includes the mass-effect of the tumor as well as that of peri-tumor edema. Most of the mass-effect of many real tumors is attributed, not to the bulk tumor itself, but to the peri-tumor edema [7. Additionally, the model employs a non-linear constitutive material model for brain tissues and a non-linear FE formulation which allow the simulation of realistic, large-deformation tumor cases. The details of the approach are presented in Sect. 2.

Another contribution of this work is the quantitative comparison of model's predictions to deformations caused by real tumors and observed in a dataset of serial MRI scans. This dataset is composed of brain images of a brain tumor patient, and uniquely available scans of three canines with surgically transplanted tumors. Results reported in Sect. 3 show that the presented model can reproduce a large percentage of the deformations caused by the real tumors. The paper is concluded with a discussion of the results in Sect. 4.

\section{Methods}

The aim of the proposed model is to simulate only the mass-effect component of tumor growth via a mechanical FE model constructed from 3D medical images. Since tumor growth is not purely a mechanical process, but involves a host of interacting biological, biochemical and mechanical mechanisms, it is essential to initialize the model simulations with a configuration for the brain from which the target configuration (that deformed by the tumor at the desired stage of tumor growth) is reachable by solving a contiuum mechanics problem.

The proposed approach can be understood by aid of Fig. 1. Let $\kappa_{o}$ be the initial configuration of the brain before the tumor emergence. The stresses in $\kappa_{O}$ are assumed negligible. Let $\kappa_{t}$ be the configuration of the brain at the target stage of tumor development. The bulk tumor denoted by $T_{t}$, is assumed to be 


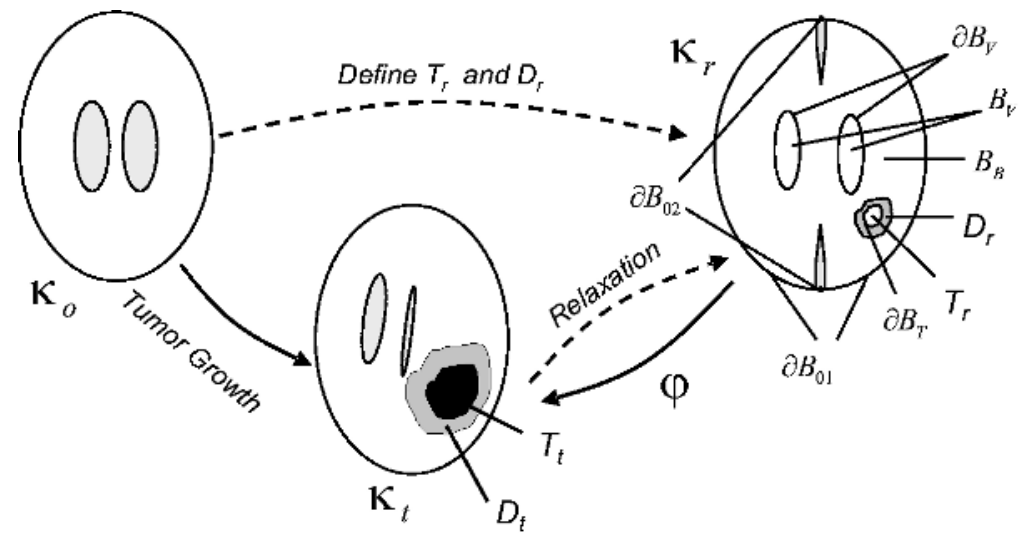

Fig. 1. A schematic showing the three configurations involved in the model. $\kappa_{o}$ is the brain before tumor development, $\kappa_{t}$ is brain at the desired stage of tumor growth, and $\kappa_{r}$ is the corresponding relaxed configuration. $T_{t}$ and $D_{t}$ are the bulk tumor and peritumor edema regions in $\kappa_{t}$ respectively, while $T_{r}$ and $D_{r}$ are the corresponding regions in $\kappa_{r}$. In $\kappa_{r}$, the ventricles are denoted by $B_{V}$, and $\partial B_{01}$ denotes the outer surface of the brain except for $\partial B_{02}$ where the falx meets the skull.

composed of proliferative, quiescent and necrotic tumor cells [6]. A region $D_{t}$ of brain tissue swollen by edema may also be associated with the tumor.

If $T_{t}$ were resected, the edema were diffused, and the stresses were allowed to relax, brain tissues will reach a relaxed configuration $\kappa_{r}$. There is a relaxed configuration associated with every $\kappa_{t}$ and it is, in general, different from both $\kappa_{t}$ and $\kappa_{o}$. Given $\kappa_{r}$, the stresses caused by the bulk tumor, and the swelling due to edema, the deformation map $\varphi$ can be obtained by solving the mechanics problem described below. For real tumor cases, these parameters are not known, but they can be estimated from the measured pattern of deformed anatomy as described [5]. For simulating mass-effect of tumors starting with normal brain images, approximations of these parameters are used as explained next.

Defining $\kappa_{r}$ involves specifying the geometry of the brain and that of $T_{r}$ (which corresponds to brain tissue that is no longer present in $\kappa_{t}$ ) and $D_{r}$ (which corresponds to brain tissue that is swollen by edema in $\kappa_{t}$ ). These regions are highly variable for different tumor cases and types. For tractability, herein, we will consider the approximation of $T_{r}$ and $D_{r}$ with two concentric spheres. Since expansion due to edema happens mainly in white matter (WM) [7, 8, $T_{r}$ is restricted to WM tissues. The center and radii of $T_{r}$ and $D_{r}$ are treated as model parameters. It is worth noting that the shape of the final tumor depends on the generated surrounding stresses and need not be spherical [1.

To account for the mass-effect of the bulk tumor, we follow the work of Wasserman and Acharya [6] and assume that the expansive force of the neoplasm can be approximated by a constant outward pressure $P$ acting on the tumor boundary. $P$ is a parameter that determines the mass-effect of the bulk tumor, and therefore, to a large extent, the final tumor size. 
Edema expansion in WM is mostly perpendicular to the direction of the fibers [7, 8. Here, we assume no knowledge of WM fibers' orientation and an isotropic expansive strain $e$ is applied to $D_{r}$ by using analogy to thermal expansion. Studies of brain edema that measured a volume expansion of $200 \%-300 \%$ in WM [7,8 imply $e \in[0.26,0.44]$. For simulations starting with normal brain scans, a value of $e=0.35$ is adopted.

\section{Continuum Mechanics Problem Statement}

Given the time scale of the tumor growth process, the deformation of brain tissues may be modeled as a quasi-static process. Additionally, if the effect of gravity is ignored, the required deformation map $\varphi: \kappa_{r} \rightarrow \kappa_{t}$ can be found by solving the static equilibrium equation $\operatorname{Div}(\boldsymbol{S})=0$, where $\boldsymbol{S}$ is the first PiolaKirchhoff tensor which is related to strain via the material constitutive law 9 .

Based on simulations using several of material constitutive laws for brain tissues suggested in the literature, we adopted the isotropic and homogeneous hyperelastic model proposed by Miller and Chinzei 10 while relaxing the perfect incompressibility assumption and ignoring viscous effects (since the time involved in tumor growth is much larger than the viscous time constants). Under these conditions, the strain energy density function of the material becomes [9]:

$$
W=\frac{2 \mu}{\alpha^{2}}\left(\bar{\lambda}_{1}{ }^{\alpha}+\bar{\lambda}_{2}{ }^{\alpha}+\bar{\lambda}_{3}{ }^{\alpha}-3\right)+\frac{1}{D_{1}}\left(J / J^{t h}-1\right)^{2},
$$

where $\bar{\lambda}_{i}=J^{-1 / 3} \lambda_{i}, \lambda_{i}, i=1,2,3$ are the principal material stretches, $J=$ $\operatorname{det}(\boldsymbol{F})$ is the volume ratio, $\boldsymbol{F}$ is the deformation gradient, $J^{\text {th }}=\left(1+e^{t h}\right)^{3}$ is the thermal volume ratio, and $e^{t h}$ is the thermal strain. The constants $\mu, D_{1}$ are related to the Young's modulus at zero strain $E_{o}$, and Poisson's ratio $\nu$ by

$$
\mu=\frac{E_{o}}{2(1+\nu)} \quad \text { and } \quad D_{1}=\frac{6(1-2 \nu)}{E_{o}} .
$$

The value $\alpha=-4.7$ determined in [10] was adopted here. Since the brain biomechanics literature includes varying accounts of brain tissue compressibility and stiffness, in the experiments described below, the effects of $\mu$ and $D_{1}$ (equivalently $\mu, \nu)$ on the proposed model were investigated. The following boundary conditions (BCs) complete the statement of the problem (refer to Fig. 11):

$$
\begin{array}{rlrlrl}
\boldsymbol{\varphi}(\boldsymbol{X}) \cdot \boldsymbol{N}(\boldsymbol{X}) & =0, \boldsymbol{X} \in \partial B_{01} & & \text { and } & \boldsymbol{\varphi}(\boldsymbol{X})=\mathbf{0}, \boldsymbol{X} \in \partial B_{02} \\
e^{t h}(\boldsymbol{X}) & =e, \boldsymbol{X} \in D_{r} & & \text { and } & e^{t h}(\boldsymbol{X})=0, \boldsymbol{X} \in B_{B} \\
\boldsymbol{S} \boldsymbol{N}(\boldsymbol{X}) & =P J \boldsymbol{F}^{-T} \boldsymbol{N}(\boldsymbol{X}), & \boldsymbol{X} \in \partial B_{T} & \\
\boldsymbol{S N}(\boldsymbol{X}) & =\mathbf{0}, & & \boldsymbol{X} \in \partial B_{V} &
\end{array}
$$

where $\boldsymbol{N}(\boldsymbol{X})$ is the outward surface normal at $\boldsymbol{X}$ in the relaxed configuration. Equation (3) implies a sliding BC over the brain surface except for locations where the falx meets the inner surface of the skull which are assumed pinned [1]. 
Equation (41) implies that the expansive strain due to edema is restricted to $D_{r}$. Equation (5) is the traction BC implied by the tumor pressure, expressed in terms on normals in $\kappa_{r}$. The ventricles are assumed void and eqn. (6) implies negligible intra-ventricular pressure [1].

\section{Experiments and Results}

Here, we provide partial validation results for the model by quantitatively comparing its predictions to the deformations observed in four brain tumor cases. The same dataset is used to guide the selection the material model parameters. Finally, a model simulation on an MRI of a normal subject is demonstrated.

Assuming that canine brain tissue properties and tumor growth process are reasonably representative of their counterparts in humans, three of the studied tumor cases were for dogs with surgically transplanted glioma cells [12] (DC1, DC2, DC3). A baseline scan was acquired before tumor growth, followed by scans on the $6^{\text {th }}$ and $10^{\text {th }}$ day post-implantation. Gadolinium-enhanced T1 MR images were acquired (MPRAGE for DC1, DC2). Tumors grow rapidly to a diameter of $1-2 \mathrm{~cm}$ by the $10^{\text {th }}$ day on which the animals were sacrificed, and prior to the presentation of significant neurological complications. The fourth dataset (HC) comes from a series of T1 MRI scans of a human with a low-grade glioma transforming into malignancy. Two scans were used with approximately 2.5 years in between. Increase in the tumor mass and significant swelling due to edema were observed. The dataset is described in Tab. 11 and example images are shown in Fig. 2,

To compare the model predictions to actual deformations in the available datasets, values of the model parameters (center, radii of $T_{r}$ and $D_{r}$, and $P$ ) for these cases must be determined. To avoid optimizing all parameters for each tumor case, the first images after tumor development $\left(6^{\text {th }}\right.$ day scan for dog cases, and the first scan for the HC) were used to approximate $\kappa_{r}$. This approximation involves the assumption of negligible edema spread and tumor infiltration

Table 1. Description of the image scans and results for the dog cases (DC1, DC2, DC3) and the human case (HC). Optimal values of the model parameters $P$ and $e$ for $\mu=842 \mathrm{~Pa}$ and $\nu=0.485$ are provided. The number of landmarks, landmark deformation statistics (lmrk def: mean/max/std. dev.) and model residual errors (Error, mean $/ \mathrm{max} / \mathrm{std}$. dev.) for the landmark points are reported.

\begin{tabular}{|l|c|c|c|c|}
\hline & DC1 & DC2 & DC3 & HC \\
\hline Image dimensions & $256 \times 256 \times 100$ & $256 \times 256 \times 100$ & $256 \times 256 \times 124$ & $256 \times 256 \times 124$ \\
\hline Voxel size, $m m$ & $0.39 \times 0.39 \times 0.7$ & $0.39 \times 0.39 \times 0.7$ & $0.47 \times 0.47 \times 1.0$ & $0.94 \times 0.94 \times 1.5$ \\
\hline$P, \mathrm{~Pa}$ & 8000 & 7000 & 15000 & 8000 \\
\hline$e$ & 0.3 & 0.15 & 0.4 & 0.3 \\
\hline Num. landmarks & 25 & 21 & 20 & 21 \\
\hline lmrk def, $m m$ & $2.16 / 3.9 / 1.06$ & $1.77 / 2.83 / 0.58$ & $1.82 / 3.04 / 0.78$ & $4.53 / 6.09 / 0.9$ \\
\hline Error, $m m$ & $1.11 / 2.5 / 0.73$ & $1.13 / 2.1 / 0.42$ & $1.19 / 2.24 / 0.52$ & $1.7 / 3.09 / 0.77$ \\
\hline
\end{tabular}



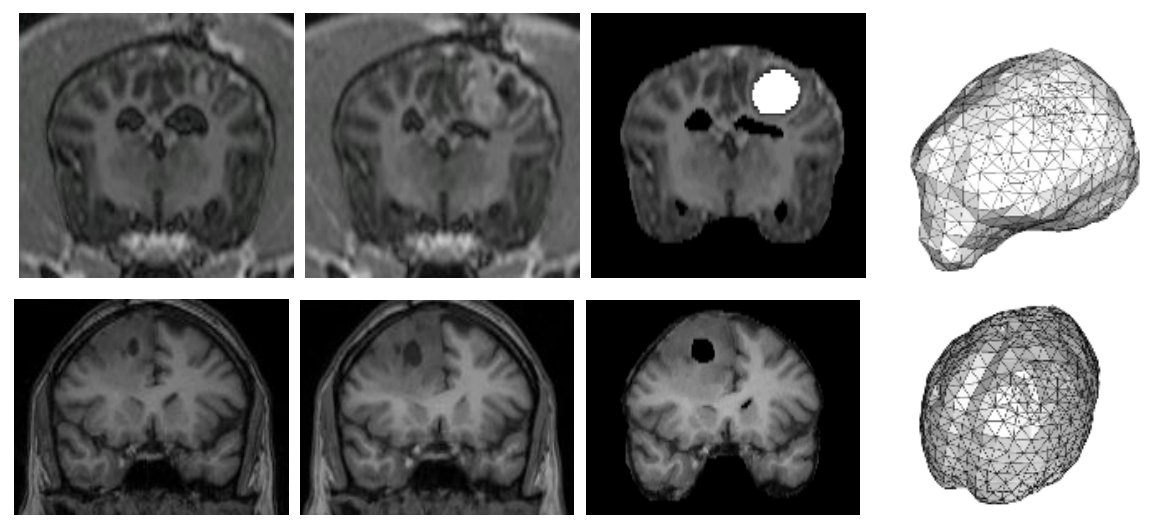

Fig. 2. Example cross sectional images from the starting (left column) and target (middle left column) 3D images for DC1 (upper row) and HC (lower row) compared to the deformed images obtained via the optimal model parameter values (middle right column). Tumors in simulated images are assigned similar intensities to the real images. The right column shows the outer surface of the used FE meshes.

between this scan and final scan, which corresponds to $\kappa_{t}$. Additionally, since tumors in the starting images are small, the stresses and deformation are assumed to be negligible. Under these assumptions, $D_{r}$ and $T_{r}$ are obtained from segmentations of the tumor and edema in the starting images. Since the starting images already had some edema, $e$ was treated as a parameter with $e \in[0.1,0.4]$.

Since loads in the proposed model are in the form of a pressure, $P$, and a prescribed strain $e$, the resulting deformation depends on $e$ and the ratio $P / E_{o}$, but not $P$ alone (confirmed by actual simulations). Given this, the value of $\mu=842 \mathrm{~Pa}$ suggested in [10] was chosen. Experiments were performed to determine $\nu$, and $P$, $e$ for each tumor case according to the following procedure.

First, rigid registration of the target (final) scan to the respective starting scan (used to approximate $\kappa_{r}$ ) was performed [13]. At least, 20 pairs of corresponding landmarks were manually identified by a human rater in the starting and target images. The landmarks were selected near the tumor, where large deformation occurs. A combination of manual and automatic segmentation of the starting images into brain, ventricles, falx, tumor and edema was then performed. A tetrahedral FE mesh was generated from the segmented images [14, and for each value of $P, e$, and $\nu$, the FE simulation environment ABAQUS [9] was used to solve the continuum mechanics problem described above. The resulting deformation map $\varphi$ for each simulation was used to deform the starting images and the locations of the landmark points in those images. Errors between deformed landmark coordinates and the corresponding rater's coordinates in the target scan were computed.

Experiments with different values of $\nu, P, e$ were performed in the following sequence. With $\nu=0.49$ [11] (which implies $E_{o}=2109 P a, D_{1}=4.75 e^{-5} P^{-1}$ ), $e$ and $P \in[1,16] K P a$ were varied for each case, the mean error in model predic- 

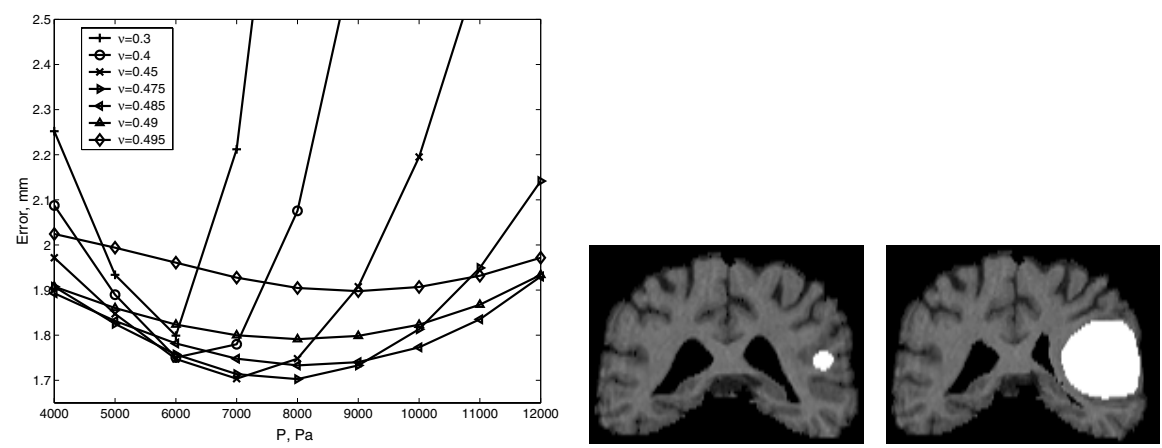

Fig. 3. Left: Mean residual errors between model predictions and deformed landmark points for $\mathrm{HC}$ for different values of $P$ and $\nu$ with $e=0.3, \mu=842 P a$. Right: $2 \mathrm{D}$ slices through a T1-weighted MRI of a normal subject before and after simulation (radius of $T_{r}$ was $5 \mathrm{~mm}, P=9 k P a$ and no edema). The simulated tumor volume is $37 \mathrm{cc}$.

tions was computed, and the value of $e$ for minimum error was recorded. With the value of $e$ determined for each case, simulations were then run for $\nu \in[0.3,0.499]$ and $P \in[1,16] K P a$. The minimum mean error occurs for $\nu \in[0.475,0.499]$ for all cases, which supports that the brain tissue is almost incompressible. We adopt the value $\nu=0.485$ - near the middle of this range. With this value of $\nu$ and $e$ determined above for each case, the optimal value of $P$ was found. Final values of $P$ and $e$ and the corresponding residual errors in the model predictions are reported in Tab. 1. The residual errors for some individual landmark points were down to $15 \%$ of the deformation observed. For $\mathrm{HC}$, the model is able to predict more than $62 \%$ of the deformation, on average. Simulated images using the optimal values of all parameters are compared to the real ones in Fig. 2, Due to space limitations, we provide an example of the error curves for only for $\mathrm{HC}$ for different values of $\nu$ in Fig. 3 where we also present the result of applying our model on a normal T1-weighted brain MRI.

To quantify the accuracy of the rater's placement of landmarks in the target image scans, for DC1, landmarks in the target image were found by two raters. The mean inter-rater variability in this case was $1.12 \mathrm{~mm}$ with a maximum of $3.29 \mathrm{~mm}$ which are similar to the respective values obtained for residual model errors. In fact, the distribution of the inter-rater distances and the average residual errors (over the two raters) were statistically indistinguishable $(p$-value $=0.95)$.

\section{Discussion and Conclusions}

A 3D FE model for simulating brain tumor mass-effect due to the bulk tumor and edema was presented. Comparison between the predictions of the model to deformations caused by four real brain tumors was performed. The results indicate that the residual errors are mostly caused by inaccuracies in the rater's 
tracking of the landmark points, as well as other errors such as modeling assumptions, errors in rigid registration and manual segmentation. In particular, in experiments on the real tumor cases, the use of segmentations of images at initial stages of the tumor to approximate the relaxed configuration makes the model unable to account for increase in infiltration and edema extent. Increase in tumor infiltration, particularly for the aggressive dog glioma tumors, may also be the reason behind perceived compressibility of brain tissues in these cases.

Future work includes investigating the relationship between model parameters and clinical variables, such as tumor type and grade. While cases studied in this paper did not involve contact between opposite walls of the ventricles, we plan to investigate the modeling of this phenomenon by using a FE contact condition, or filling the ventricles with a material that is softer than the brain tissues. The use of the presented model in establishing registration between normal brain images is presented in [5].

\section{Acknowledgments}

The authors would like to thank Dr. Nick Fox at the University College London, UK for providing the tumor patient's images, Dr. James Anderson, Carolyn Magee, Dr. Stelios Kyriacou at the Johns Hopkins University School of Medicine for preparing the dog tumor datasets. This work was supported in part by the National Science Foundation under Engineering Research Center grant EEC9731478, and by the National Institutes of Health grant R01NS42645.

\section{References}

1. Kyriacou, S.K., Davatzikos, C., Zinreich, S.J., Bryan, R.N.: Nonlinear elastic registration of brain images with tumor pathology using a biomechanical model. IEEE Transactions on Medical Imaging 18 (1999) 580-592

2. Mohamed, A., Kyriacou, S.K., Davatzikos, C.: A statistical approach for estimating brain tumor induced deformation. Proc. IEEE Workshop on MMBIA (2001) 52-59

3. Dawant, B.M., Hartmann, S.L., Pan, S., Gadamsetty, S.: Brain atlas deformation in the presence of small and large space-occupying tumors. Computer Aided Surgery 7 (2002) $1-10$

4. Cuadra, M.B., Pollo, C., Bardera, A., Cuisenaire, O., Villemure, J.G., Thiran, J.P.: Atlas-based segmentation of pathological MR brains using a model of lesion growth. IEEE Transactions on Medical Imaging 23 (2004) 1301-1314

5. Mohamed, A., Shen, D., Davatzikos, C.: Deformable registration of brain tumor images via a statistical model of tumor-induced deformation. In: Proc. of MICCAI 2005. (2005)

6. Wasserman, R., Acharya, R.: A patient-specific in vivo tumor model. Mathematical Biosciences 136 (1996) 111-140

7. Kuroiwa, T., Ueki, M., Suemasu, H., Taniguchi, I., Okeda, R.: Biomechanical characteristics of brain edema: the difference between vasogenic-type and cytotoxictype edema. Acta Neurochir. Suppl. 60 (1994) 158-161 
8. Nagashima, T., Tamaki, N., Takada, M., Tada, Y.: Formation and resolution of brain edema associated with brain tumors. a comprehensive theoretical model and clinical analysis. Acta Neurochir. Suppl. 60 (1994) 165-167

9. Hibbitt, Karlsson, and Sorensen, Inc., USA: Abaqus version 6.4. (2003)

10. Miller, K., Chinzei, K.: Mechanical properties of brain tissue in tension. Journal of Biomechanics 35 (2002) 483-490

11. Miga, M., Paulsen, K., Kennedy, F.E., Hartov, A., Roberts, D.: Model-updated image-guided neurosurgery using the finite element method: Incorporation of the falx cerebri. In: MICCAI 1999. (1999)

12. Wodinsky, I., Kensler, C., Roll, D.: The induction and transplantation of brain tumors in neonatal beagles. Proc. Am. Assoc. Cancer Res. 10 (1969)

13. Studholme, C., Hill, D.L.G., Hawkes, D.J.: An overlap invariant entropy measure of 3D medical image alignment. Pattern Recognition 32 (1999)

14. Mohamed, A., Davatzikos, C.: Finite element mesh generation and remeshing from segmented medical images. In: Proc. of the 2004 IEEE ISBI, Arlington, VA (2004) 